\title{
Erratum to: Novel method for genotyping clinical herpes simplex virus type 1 isolates
}

Brigitte Glück $^{1} \cdot$ Susanne Möbius $^{1} \cdot$ Florian Pfaff $^{1} \cdot$ Roland Zell $^{1}$. Andreas Sauerbrei ${ }^{1}$

Published online: 18 September 2015

(c) Springer-Verlag Wien 2015

\section{Erratum to: Arch Virol}

DOI 10.1007/s00705-015-2568-y

Unfortunately, Fig. 1 was published incorrectly in the original publication of the article. The correct version of Fig. 1 is given below:

The online version of the original article can be found under doi:10.1007/s00705-015-2568-y.

Andreas Sauerbrei

andreas.sauerbrei@med.uni-jena.de

Institute of Virology and Antiviral Therapy, German

Consulting Laboratory for HSV and VZV, Jena University

Hospital, Friedrich Schiller University Jena, Hans-Knöll-Str.

2, 07745 Jena, Germany 


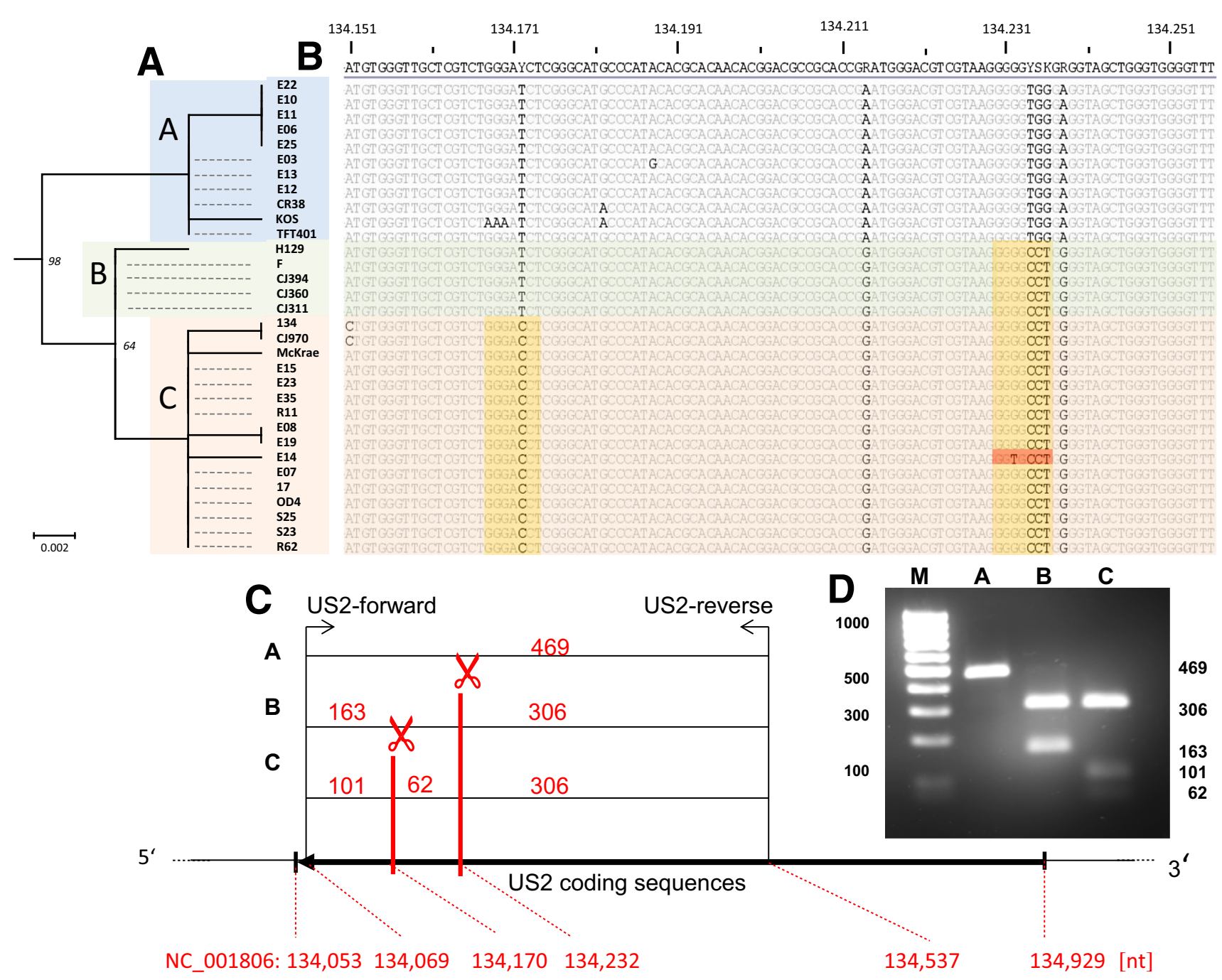

Fig. 1 Phylogenetic analysis of US2 as a basis for a novel HSV-1 genotyping method. (A) Phylogenetic tree constructed from 32 aligned amino acid sequences of HSV-1 US2 from NCBI GenBank (MEGA6 software using maximum-likelihood, the JTT-model and 500 bootstrap replicates). Three groups with high bootstrap support (italic letters) were identified and designated as A (blue), B (green) and $\mathrm{C}$ (ochre). (B) 105-nt window of the aligned coding sequence from US2 highlighted according to the group segregation of (A) and EcoO109I recognition sites (highlighted in golden colour). A single polymorphism in the EcoO109I recognition site was observed for strain E14 (highlighted in red). (C) Schematic illustration (forward primer starts at 134,069 and reverse primer ends at 134,537) and (D) results of agarose gel electrophoresis of the three HSV-1 US2 genotypes based on RFLP analysis of an amplified 469-bp DNA fragment using EcoO109I (ঝ). Genotype A, no cleavage, 469-bp fragment; genotype B, cleavage into 306-bp and 163-bp fragments; genotype C, cleavage into 306-bp, 101-bp and 62-bp fragments 